\title{
Denaturing Gradient Gel Electrophoresis (DGGE): A Rapid and Sensitive Technique to Screen Nucleotide Sequence Variation in Populations
}

BioTechniques 27:1016-1030 (November 1999)

\author{
Kristina M. Miller, Tobi J. \\ Ming, Angela D. Schulze and \\ Ruth E. Withler \\ Pacific Biological Station, \\ Department of Fisheries and \\ Oceans, Nanaimo, BC, Canada
}

\section{ABSTRACT}

We describe a rapid and sensitive method for the detection of nucleotide sequence variation that can be used for largescale screening of population markers. Denaturing gradient gel electrophoresis (DGGE) detects sequence variants of amplified fragments by the differences in their melting behavior. DGGE detects most single-base substitutions when carried out on products amplified with a primer to which a GC clamp has been added. Although $D G G E$ has been primarily used for the detection of limited numbers of single-base mutations in disease studies, it offers great potential for use in population analysis of genetic markers with greater levels of sequence variation. The methodology described was developed to identify the number and distribution of MHC class I $\alpha 1$ alleles among chinook salmon (Oncorhynchus tshawytscha) populations. DGGE detects 28 of 31 identified $\alpha 1$ sequences, which differ by between 1 and $16 \mathrm{nu}$ cleotides and a two-codon indel. By creating a network of control alleles, 22-23 of the MHC alleles can be resolved rapidly and accurately by a single gel run condition, and 27 alleles can be resolved by two gel run conditions. This technique has been used in surveys scoring alleles from two MHC markers (class I $\alpha 1$ and $\alpha 2$ ) in 20000 individuals of chinook and coho (O. kisutch) salmon. A single person in our laboratory now analyzes 160 salmon from one MHC locus per day with DGGE.

\section{INTRODUCTION}

An increasing ability to survey DNA polymorphisms without resorting to laborious sequencing techniques has touched off explosive growth in studies of genes and the populations that carry them. Progressively more of the variation present in DNA sequences became detectable as the technology advanced from starch gel electrophoresis of allozymes through Southern blotting and restriction fragment-length polymorphism (RFLP) analysis of extracted mitochondrial, ribosomal and minisatellite DNA. The advent of polymerase chain reaction (PCR) allowed an even larger array of mitochondrial and nuclear loci to be examined with much greater sensitivity.

For population surveys, acrylamide gel electrophoresis of variation at microsatellite loci, which are non-coding and mutate rapidly, has become the technique of choice. The apparent neutrality of allelic variants at these loci, and the ability to quickly identify alleles by length, make them ideal for studies of population structure resulting from the restriction of gene flow and the stochastic effects of limited population size. However, they provide little information on the subspecific processes of adaptation and speciation occurring as the result of natural selection acting upon polymorphism at coding loci.

PCR-amplified loci with nucleotide (nt) sequence variation have been studied in populations, but the techniques used to detect sequence variation are neither as fast nor as sensitive as those applied to length variation. Direct DNA sequencing is the most sensitive technique, but even with an automated sequencer is too labor-intensive for large- scale population surveys. RFLP screening, widely used in population studies, only samples a subset of the sequence variation in amplified fragments and generally requires a large battery of enzymes. Single-stranded conformational polymorphism (SSCP) analysis reveals polymorphism over the entire fragment length by detecting the mobility change of single-stranded (ss)DNA in polyacrylamide gel electrophoresis (PAGE) resulting from its altered folded structure (7). SSCP is fairly sensitive and widely used for population analysis, although most studies use radioactivity.

Denaturing gradient gel electrophoresis (DGGE) is nonradioactive and uses the difference in melting behavior of double-stranded (ds)DNA induced by nucleotide sequence variation to differentiate alleles. DGGE has been used primarily for detection of specific mutations in disease studies $(5,22)$, phylogenetic analysis of bacterioplankton (14) and separation of alleles for direct sequencing $(1,9)$. Our studies suggest that DGGE also has great potential for identification of alleles in population surveys $(10,11)$. DGGE is extremely sensitive, detecting over $99 \%$ of single-nucleotide sequence variation (19), and rapid, with PCR products loaded directly onto polyacrylamide denaturing gradient gels. DNA fragments between 50 and $500 \mathrm{bp}$ in length, preferably with a single melting domain, are most suitable for analysis with DGGE. For DNA fragments with two or more melting domains, the addition of a GC clamp to the end with the higher melting temperature $\left(\mathrm{T}_{\mathrm{m}}\right)$ is required to detect base pair substitutions in the high melting domain (19).

In DGGE, amplified dsDNA fragments are electrophoresed through a gel containing a gradient of chemical 
(urea and formamide) denaturant (3). When a fragment reaches its denaturing point in the gel, it begins to dissociate and its migration is retarded. The difference of even a single nucleotide between fragments will alter their relative melting behaviors, allowing detection using DGGE. Because a gradient of denaturant is used, fragments with a single melting domain and a range of denaturing points can be differentiated on a single gel run. This makes the time required for the detection of sequence variation at a highly polymorphic coding locus comparable with that required for sampling length variation at a single microsatellite locus. Alternatively, fragments with two melting domains may require two gel run conditions to adequately sample both domains.

In this study, we describe a DGGE methodology developed to identify the number and distribution of allelic sequence variants at a major histocompatibility complex (MHC) locus within and among chinook salmon (Oncorhynchus tshawytscha) populations of British Columbia, Canada. MHC exons with protein-binding regions encode antigenic proteins that enable vertebrate organisms to distinguish self from non-self and are the most polymorphic coding loci in vertebrate species (16). The extremely high levels of polymorphism at MHC loci are maintained by balancing and/or frequency-dependent selection, and linkage relationships between specific MHC haplotypes and disease resistance have been established (20). A low level of MHC polymorphism has been used as an indicator of reduced adaptive variation resulting from population bottlenecks $(8,18)$. However, large surveys of MHC variation have been hampered by the laborious or insensitive methods available to detect nucleotide sequence variation.

In a study of MHC class I $\alpha 1$ sequence variation among 40 fish from two chinook salmon populations, our single locus primers revealed 22 alleles differing from one another by 1-16 nt plus a two-codon indel (10). We used these sequence data to design a DGGE mutation detection system that could rapidly identify most of the alleles (and 13 additional alleles discovered during the study) in a large-scale survey of MHC genotypes in chinook salmon populations. The need to detect nucleotide substitutions in both high and low melting domains of the $\alpha 1$ exon resulted in the possibility that alleles would shift relative positions under different run times. Thus, a system of standards was required to monitor the migration of known alleles and detect the presence of new ones. We needed the ability to incorporate new alleles, as they were discovered, into the standard set of known alleles. In this study, we outline the development and application of our DGGE system and demonstrate its use in the consistent scoring of chinook salmon MHC $\alpha 1$ sequence variants over time and across many gels. We also describe how the system was used to identify MHC $\alpha 1$ alleles in the closely related coho salmon $(O$. kisutch), for which little sequence data was available before the undertaking of a population survey.

\section{MATERIALS AND METHODS}

\section{PCR and the GC Clamp}

DNA was extracted from tissue samples of adult chinook salmon sampled from rivers in British Columbia, Canada. Both frozen liver tissue (12) and operculum/fin clips (21) preserved in $95 \%$ ethanol were used. Primers specific to the MHC class I $\alpha 1$ exon homologous to the classical Sasa-p30 locus $(6,13)$ were used to amplify fragments of 219-225 bp (excluding primers). Known sequences for $22 \alpha 1$ alleles determined in a previous study indicated that the majority of nucleotide sequence variation between alleles resides on the $3^{\prime}$ end of the $\alpha 1$ exon (10). The melting curves of these sequences determined by WinMelt ${ }^{\mathrm{TM}}$ software (Bio-Rad, Hercules, CA, USA) showed that two melting domains were present, with the $5^{\prime}$ end of the $\alpha 1$ exon melting at a higher temperature than the $3^{\prime}$ end. To facilitate sampling of variation in both high and low melt domains and to maximize the resolution of the highly variable $3^{\prime}$ end of the fragment, a GC clamp was added to the (high melt) $5^{\prime}$ end of the amplified $\alpha 1$ fragment. The $5^{\prime}$ primer sequence $\left(\mathrm{A} 1 \mathrm{R}_{\mathrm{CL}}\right)$ containing the 41-bp GC clamp (in bold), was 5'-GCCCGCCC-
CCGCGCCCTGCCCGCGCCCCGCGCCGCCCGCCCTGACTCACGCCCTGAAGTA-3', and the 3' primer sequence (A1EF) was 5'-CTCCACTTTGGTTAAAACG-3'. PCR was carried out in a total volume of $50 \mu \mathrm{L}$ containing $0.2-0.5 \mu \mathrm{g}$ of DNA, 15 pmol of each primer, $200 \mu \mathrm{M}$ of each dNTP and $1.3 \mathrm{U}$ of Taq DNA Polymerase (PE Biosystems, Foster City, CA, USA). The profile $\left(94^{\circ} \mathrm{C}\right.$ for $1 \mathrm{~min}, 51^{\circ} \mathrm{C}$ for 2 min and $72^{\circ} \mathrm{C}$ for $2 \mathrm{~min}$ at 35 cycles) amplified one or two fragments per individual, suggesting that these primers amplify alleles from a single locus. This supposition was confirmed by pedigree analysis of 17 families of coho salmon (unpublished data).

\section{DGGE}

Perpendicular DGGE. DGGE was performed using the $\mathrm{D} \mathrm{Gene}^{\mathrm{TM}}$ or $\mathrm{D}$ Code $^{\mathrm{TM}}$ electrophoresis systems (both from Bio-Rad). For both perpendicular and parallel DGGE, $16 \times 16 \times 0.1 \mathrm{~cm}$ gels were poured with $7.5 \%$ polyacrylamide (acrylamide:bis-acrylamide at $37.5: 1)$ in $1 \times$ TAE buffer $(40 \mathrm{mM} / \mathrm{L}$ Tris, $40 \mathrm{mM} / \mathrm{L}$ sodium acetate, $1 \mathrm{mM} / \mathrm{L}$ EDTA, pH 7.4) mixed with varying (gradient-dependent) concentrations of urea and formamide and polymerized by the addition of $0.1 \%$ TEMED and $0.1 \%$ ammonium persulfate. Denaturing gradients were formed by linearly increasing concentrations of urea/formamide (0.07 $\mathrm{M}$ urea and $0.4 \%$ formamide per $\%$ denaturant) using a gradient maker (Model 475 Gradient Delivery System; Bio-Rad). D Gene and D Code systems contained $7 \mathrm{~L}$ of $1 \times$ TAE buffer at $\mathrm{pH}$ 7.4 and heated to $56^{\circ} \mathrm{C}$. All reagents were of electrophoretic grade.

We determined initial DGGE conditions by electrophoresing the amplified chinook salmon $\alpha 1$ alleles on perpendicular gradient gels. Perpendicular gels, on which the chemical gradient runs perpendicular to the electrical current, contain a single long well at the top of the gel in which the amplified product of one or multiple alleles are loaded. The S-shaped curves produced by the movement of the DNA through the perpendicular denaturant gradient show the range of denaturant over which the amplified products dissociate, and fragments that can be differen- 
tiated by DGGE will have slightly shifted melting curves.

DNA amplified from TA Cloning ${ }^{\circledR}$ Vector (Invitrogen, Carlsbad, CA, USA) of $3 \alpha 1$ alleles were combined, and a sample volume of $150 \mu \mathrm{L}$ was loaded onto a perpendicular gel containing a $15 \%-90 \%$ denaturant. Gels were electrophoresed for $4 \mathrm{~h}$ at $110 \mathrm{~V}$, stained with ethidium bromide and visualized and photographed on an UV transilluminator.

Parallel DGGE. We used the 35\%$55 \%$ denaturing gradient established in the perpendicular run to examine the migration of all 22 known $\alpha 1$ alleles in parallel gradient gels. Parallel gels, on which the chemical gradient runs parallel to the electrical current, contain 30 wells at the top in which to run samples. Time series analysis of the 22 previously sequenced alleles enabled us to determine the range of denaturant that separated the most alleles (homoduplexes only) and to assess changes in the relative positions of alleles at different denaturant concentrations. On each gel, $6 \alpha 1$ alleles were loaded (10-20 $\mu \mathrm{L}$ ) into different lanes at $3 \times$ during a $7-\mathrm{h}, 120-\mathrm{V}$ run, at 0,1 and $2 \mathrm{~h}$. The effect of the different run times (from 5-7 h) was to electrophorese the alleles into different portions of the denaturing gradient. All 22 alleles, in various combinations, were run using this procedure to determine the range of denaturant within which the greatest number of alleles were differentiated and the relative positions of all 22 alleles within that range. We used results of this analysis to adjust the range of denaturant for subsequent parallel gels and then re-ran the time series analysis at $1 / 2-\mathrm{h}$ intervals to determine the appropriate run time within the adjusted range.

Standard allele sets. Once run conditions were optimized, we amplified TA clones of the 22 chinook salmon $\alpha 1$ alleles in multiple PCRs and concentrated the products to $1 / 4-1 / 2$ of their original volume with a SpeedVac ${ }^{\circledR}$ SVC-100H (Savant Instruments, Holbrook, NY, USA). Subsets of the alleles were combined into three standard sets to use as controls in the following way: the relative positions of alleles run under optimal conditions were determined from the time series analysis, and the fastest (latest melting) to slow- est (earliest melting) alleles were alternately placed into each of the three standard sets. Alleles that were known to shift relative positions at different denaturing concentrations (as identified in the time series analysis) were placed in different standard sets. Each of the standard sets and all $22 \alpha 1$ alleles were then electrophoresed on a single gel under optimal conditions to confirm that each of the allelic homoduplexes in the standard sets was visible as a distinct fragment in the standard lane, and that its position corresponded with that of the individual allele run separately. The standard sets and individual alleles were then run in a time series to enable interpretation of the changes that occurred in the standard set lanes under different denaturant concentrations. Time series analysis of the standard sets was routinely performed during the population surveys to adjust run times when new batches of acrylamide were purchased or when problems arose.

Population analysis. In the population analyses, 45\%-60\% denaturant range parallel gels were poured with 30 -well combs. Amplified $\alpha 1$ fragments of 20 individuals from one to two populations were run on each parallel gel. The allele standard sets were loaded in three adjacent lanes in the center and on each side of the gel. DGGE gels were photographed at 1024 $\times 1024$ pixel density with a charge-coupled device (CCD) camera (Eastman Kodak, Rochester, NY, USA) and analyzed with BioImage WholeBand ${ }^{\mathrm{TM}}$ software (Genomic Solutions, Ann Arbor, MI, USA). The standard allele sets were used to confirm that the electrophoretic conditions were sufficient to resolve all of the known alleles, to monitor the relative positions of shifting alleles on the gel and to form a network on which to score individual alleles. Alleles within the standard lanes were assigned "DGGE allele" numbers ranging from 50-400, approximately corresponding with their relative positions on the gel. New alleles identified in the population survey with denaturing points that did not coincide with any of the known alleles were added to a standard lane as they arose.

All new alleles identified by DGGE were cloned (from one or two representative individuals) into the TA vector, and multiple clones were sequenced with a Sequenase II $^{\mathrm{TM}}$ Kit (US Biochemicals, Cleveland, OH, USA). Sequencing autoradiographs were scanned onto a Sun ${ }^{\circledR}$ Spark ${ }^{\mathrm{TM}}$ Workstation (Sun

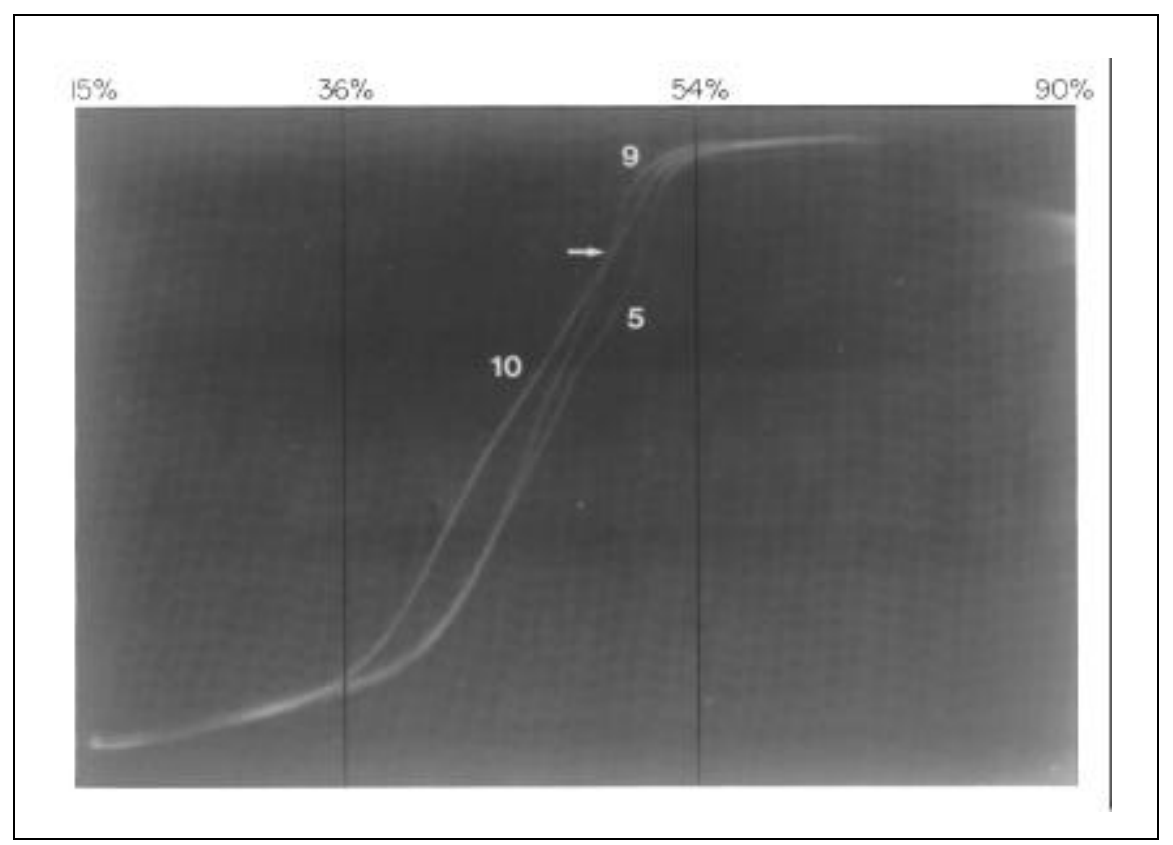

Figure 1. Perpendicular DGGE of $\alpha 1$ alleles 5,9 and 10 run on a chemical gradient of $15 \%-90 \%$. Melting curves for $\alpha 1$ alleles ranged from $36 \%-54 \%$. The crossing of the curves for alleles 9 and 10 (arrow) suggests that two melting domains are present, with the low melting domain dissociating between $36 \%$ and $51 \%$ denaturant and the high melting domain dissociating between $51 \%$ and $54 \%$ denaturant. 


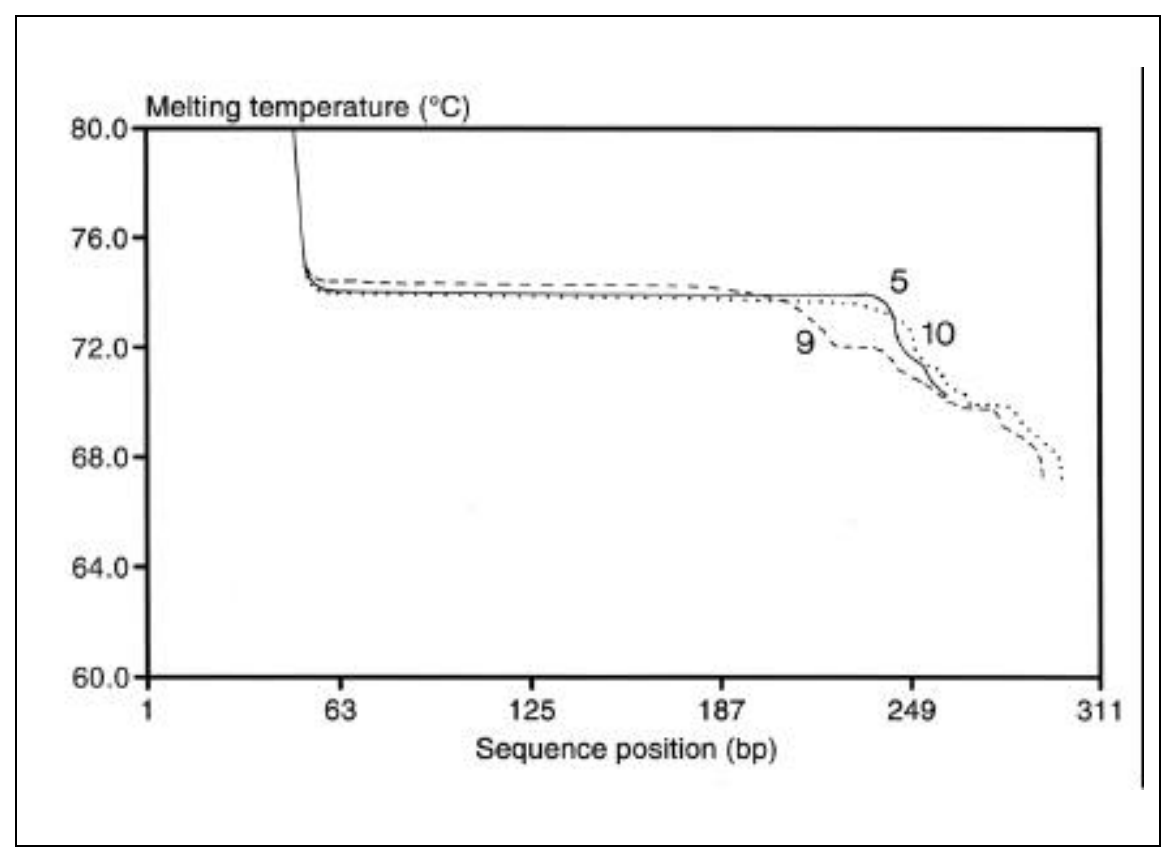

Figure 2. Predicted melting curves for alleles 5, 9, and 10 calculated using WinMelt software. The predicted melting curve includes the $60-\mathrm{bp} A 1 \mathrm{R}_{\mathrm{CL}}$ primer sequence.
Microsystems, Palo Alto, CA, USA) using a Scanmaster ${ }^{\circledR} 3+$ (Howtek, Hudson, NH, USA) and were read and aligned using BioImage software.

\section{RESULTS}

\section{Perpendicular DGGE}

The melting curves from perpendicular DGGE of alleles 5, 9 and 10 were $\mathrm{S}$ shaped, with a range of $36 \%-54 \%$ and the midpoint at $47 \%$ (Figure 1). All three chinook salmon alleles were resolved on the perpendicular gel, but the curves of alleles 9 and 10 crossed at $51.5 \%$ denaturant, indicating that allele shifting might occur in different portions of the denaturing range. Alleles 5 and 10 were separated throughout the entire denaturant range, while alleles 5 and 9 separated at denaturant concentrations of $45 \%$ and higher. 


\section{WinMelt Software Analysis}

Analysis of the melting behavior of the $\alpha 1$ fragments with the WinMelt software resolved two factors that could cause shifting of alleles. One, a second lower melting domain was apparent at the $3^{\prime}$ end of the fragments, opposite the GC clamp, starting at nt 185 (Figure 2 ). Thus, the placement of the GC clamp on the $5^{\prime}$ end of the $\alpha 1$ fragment was not sufficient to create a single melting domain over the entire fragment. WinMelt analysis of fragments containing the GC clamp on the $3^{\prime}$ end also revealed two melting domains. Two, the predicted melting curves for alleles 10 and 9 crossed at nt 206 (nt 146 not including the clamped primer), which is the location of the two-codon indel. As allele 10 contains the insert and allele 9 does not, it appears that the
11111111111111111222

1234456722344666677777888012 590442668646112156816789789951

Onts $-\mathrm{A} * 1$

onts $-\mathrm{A} * 2$

Onts $-\mathrm{A} * 3$

Onts $-\mathrm{A}^{*} 4$

Onts $-\mathrm{A} * 7$

Onts $-A * 25$

Onts $-A * 5$

Onts $-\mathrm{A} * 31$

Onts $-\mathrm{A} * 23$

Ont $s-A * 9$

Onts $-\mathrm{A} * 21$

Onts $-A * 8$

Onts $-\mathrm{A} * 29$

Onts $-\mathrm{A} * 27$

Onts $-A * 35$

Onts $-\mathrm{A} * 10$

Onts $-\mathrm{A} * 32$

Onts $-\mathrm{A} * 30$

Onts $-\mathrm{A} * 33$

Onts $-\mathrm{A} * 16$

Onts $-\mathrm{A} * 36$

Onts $-\mathrm{A} * 20$

Onts $-A * 18$

Onts $-A * 22$

Onts $-\mathrm{A} * 15$

Onts $-\mathrm{A} * 14$

Onts $-\mathrm{A} * 26$

Onts $-\mathrm{A} * 28$

Onts $-\mathrm{A} * 12$

Onts $-\mathrm{A} * 13$

Onts $-\mathrm{A} * 11$
ACGCCCGTTGGAGC-AGACTATTTGCCGAC

.G. . . . A . . . . . . . .

$\ldots \ldots \ldots$ A. . . . . .

$\ldots \ldots$. А. . . . . . .

$\ldots$ T............. GAA.AG.

$\ldots \ldots \ldots$ A. . . . A.A.T

..T.A................ .

..T.A................ . T

.G.T.TA.............GG..A.T

.. T. . . . TAGA............. . T

..T...C.T. .AAI........GA..A.T

..T... T.A............ T

.G.T. . A.A.A.......A.A.

T.T....ATAGAAI.........A.A.T

..AT. . . TAGAAI. TGGA...GA. .A.T

. AT.... TAGAAI. .GGA...G.A.A. .

... T.... A. AAI. TGGA.....A.A. .

.G.T. . . . A.AAI . GGA . . . A.A. .

... . . . . . A.AAI. .GGA....A. .A. .

... . . . . A.AAI. .GGAT . . . . A. .

... T. . . . . AGAAI . TGGAT. GA. . . A .

... T..... AGAAI.TGGAT . GAAA. A. T

... T.... . TAGAAI. . GGATGGA... A.T

...T. . . . TAGAAI. .GGAT.GA...A.T

.. T. .AC.TA.AAI. .GGAT. .GA. A.T

T. T. . . . TA.AAI . GGAT . GA. . A.T

T. T. . . . TA. AAI . GGAT . G. . A. .

.G.T...C.TA.AAI.TGGAT..G.A.A. .

...T. . C.TA.AAI.TGGAT. .G.A.A. .

... T. . . . TA. AAI. TGGAT. .G.AAA. .

...T.... .TA.AAIGTGGAT. .G.A.A. .

Figure 3. Nucleotide sequences of the variable sites of $\alpha \mathbf{1}$ chinook alleles. Full allele sequences for alleles 1-22 are given in Reference 13 (GenBank ${ }^{\circledR}$ Accession Nos. U80272-U80293), for alleles 23-31 in Reference 10 (GenBank Accession Nos. AF104534-AF104542) and for alleles 32-36 (no 34) (GenBank Accession Nos. AF162867-AF162871). Numbering starts at the first nucleotide of the amplified fragment (excluding the primer and GC clamp), not at the beginning of the $\alpha 1$ exon. The "I" at position 142 indicates the two-codon indel (ACA CTG).

presence of the insert may cause the shifting of alleles 9 and 10.

With this knowledge, we could have redesigned our primers to amplify two fragments, one containing the high melt domain and one containing the low melt domain, which would then be analyzed by DGGE separately. Instead, we devised a method using control alleles that would allow us to monitor the relative positions of alleles for direct and accurate scoring and then ran alleles under two different run times to sample the variation in each melting domain.

\section{Parallel DGGE}

The 22 chinook salmon $\alpha 1$ alleles were initially run on $35 \%-55 \%$ parallel gradient gels. However, using this range of denaturant, alleles did not run far enough into the gel to sample the high melting domain (at approximately $50 \%-60 \%$ denaturant) during a 7-h, $120-\mathrm{V}$ run. We therefore adjusted the gel concentration to $45 \%-60 \%$, so that both the high and low melting domains could be sampled, optimally at $5.25 \mathrm{~h}$ at $120 \mathrm{~V}$ for the low melting domain and at $6 \mathrm{~h}$ at $120 \mathrm{~V}$ for the high melting domain.

The 22 chinook salmon $\alpha 1$ alleles could not all be differentiated by DGGE in the time series analyses. Alleles 2, 3 and 4, 12 and 28, 19 and 20 and 17 and 22 were completely undifferentiated from each other. Most of these sets of alleles contained an apparent G-T transversion at nt 106 of the $225 \mathrm{nt}$ fragment. WinMelt software predicted that variability at this position would result in differential melting curves that could be detected by DGGE. Subsequent resequencing of these alleles revealed that the nucleotide variation at this position (codon 44) was, in fact, a sequencing artifact due to a stop in the sequence. A G-C transition in allele 3 at nt 107 was also an artifact. After correction for this artifact, only alleles 2, 3 and 4, which differed at nt 9 (G-C transversion) and at nt 176 (A-T transversion) (Figure 3) and alleles 12 and 28, which also differed at nt 9 (G-C transversion) were undifferentiated by DGGE, also as predicted by the WinMelt software.

The failure to differentiate alleles with sequence variation close to the GC clamp (nt 9) was not surprising. In gen- 
eral, it is difficult to obtain resolution within the first $30 \mathrm{nt}$ adjacent to the clamp. Furthermore, the failure to resolve the A-T transversion at nt 176 may reflect the general relative difficulty in detecting $\mathrm{A}-\mathrm{T}$ transversions that has been cited in other studies (15).

The remaining pairs of $\alpha 1$ alleles that differed by a single nucleotide (including those subsequently identified by DGGE) were differentiated using DGGE at one or more of the run times. Alleles 31 and 5, which differed by a C$\mathrm{T}$ transition at $\mathrm{nt} 243$, alleles 11 and 12 , with a G-A transition at nt 147 and alleles 18 and 22, with a G-T transition at nt 198, were all differentiated from one another (Figures 3 and 4 and Table 1). Alleles 12 and 13, with a C-A transition at nt 189 , were slightly differentiated but ran too close together to enable consistent scoring as separate alleles.

As expected from the presence of two melting domains within the $\alpha 1$ sequence and the two-codon indel, time series analysis revealed a number of alleles that shifted relative positions on the gel when electrophoresed into different ranges of denaturant concentration. Moreover, some alleles that migrated differentially through the gel at low concentrations of denaturant became indistinguishable when electrophoresed into high denaturant concentrations (Figure 4 and Table 1). As a result, there was no single run time that consistently separated all of the alleles. Instead, each run time resolved between 21 and 23 alleles, and because the different run times were sampling variation in the two melting domains, the alleles that were resolved differed between the run times. We therefore chose to differentiate the $\alpha 1$ alleles under two run times: $5.25 \mathrm{~h}$, which resolved 22 alleles on the basis of varia- tion in the low melting domain and $6 \mathrm{~h}$, which resolved 23 alleles on the basis of variation in the high melting domain (Figure 4 and Table 1). By combining allelic resolution at both run times, we differentiated 27 of the $31 \alpha 1$ alleles (19 from our initial study [Reference 13] and 12 identified by DGGE [Reference $10])$. Only alleles with variations close to the GC-clamp $(2,3,4$ and 12, 28), with a single A-T transversion at nt 176 $(2,3,4)$ or with a single C-A transition at nt $189(12,13)$ remained unresolved.

\section{Population Analysis}

Table 1 shows the scoring of alleles under the two run times. Between the 5.25-h and 6-h run times, a number of alleles shifted relative positions and were thus scored differently under the two conditions. Examples are alleles 36 vs. 8 and 23, alleles 11 vs. 2-3-4 and al-

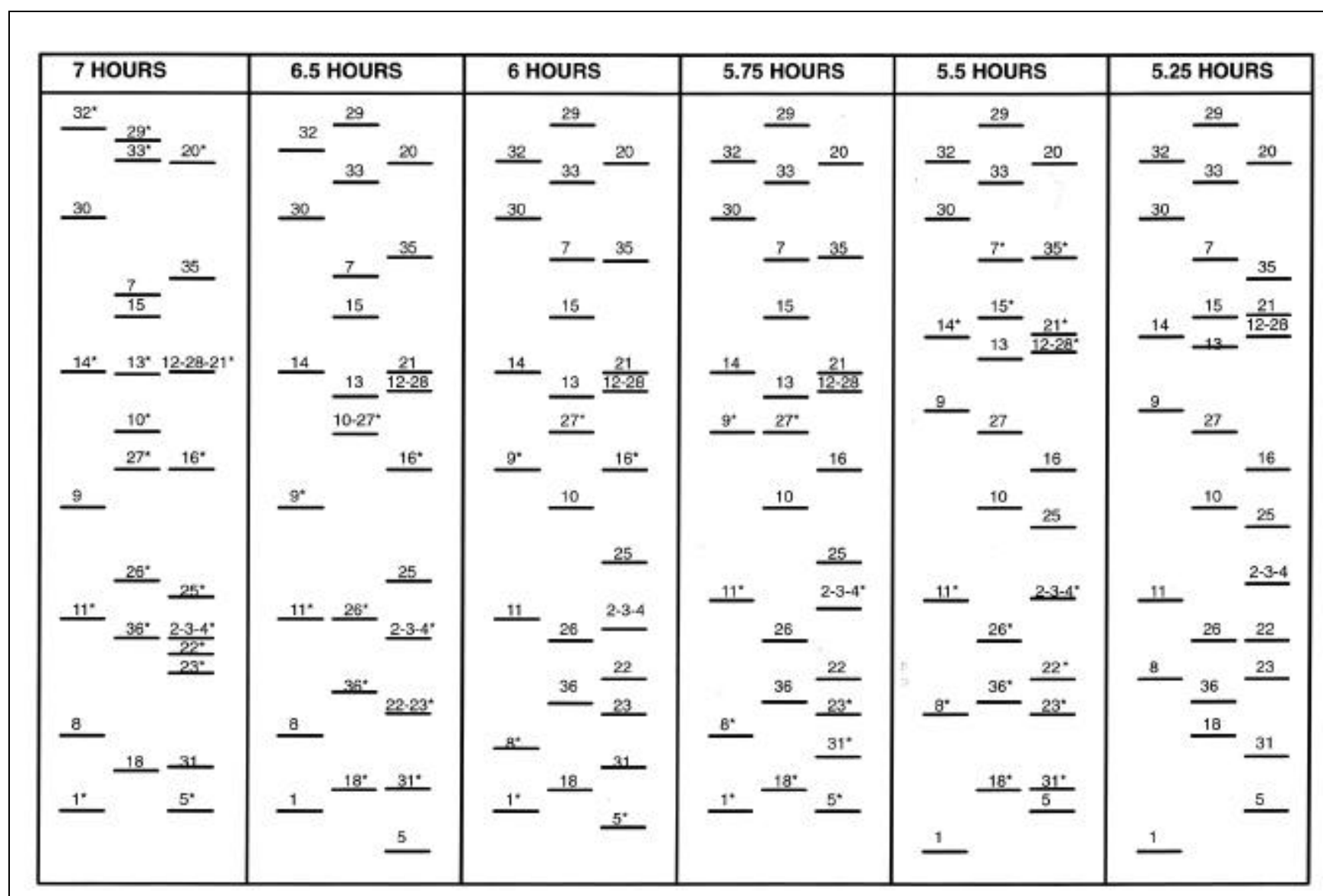

Figure 4. Schematic of time-series analysis of chinook $\alpha 1$ alleles run on a 45\%-60\% denaturant gradient for 5.25-7 h at $110 \mathrm{~V}$. Asterisks $(*)$ denote alleles that shift position from one time period to the next. The 5.25-h run time was used to sample sequence variation in the low melting domain, and the 6-h run time was used to sample variation in the high melting domain. 
leles 9 vs. 27. Other alleles that migrated to the same position under one run time were differentiated under the other. Examples are alleles 8 and 23; 22 and 26; 9 and 16;21, 15 and 14; 7 and 35 ; and 32 and 33 .

Figure 5 shows an example of a parallel DGGE gel (run time of $5.25 \mathrm{~h}$ ) used in the population surveys. The lower Fraser River population is located near the mouth of the Fraser River, while the Thompson River population is located in a tributary of the Fraser River approximately $250 \mathrm{~km} \mathrm{NE}$ from the mouth of the river. Despite the short distance between the populations, allele frequencies differ significantly, with alleles (from top to bottom) 29,32 (not in the standard), 30, 33 (not in the standard), 12, 9 and 11 found in the lower Fraser population, and alleles 20, 7 and 12 found in the Thompson River population. Note the identification of two new alleles (32 and 33, not in the standard, but later sequenced and included in this analysis).

\section{$\alpha 1$ Sequence Analysis}

There were 29 variable sites (not including a two-codon insert at codons 55 and 56) over the 219- or 225-nt chinook salmon $\alpha 1$ sequences discovered in our survey (Table 1 and Figure 3). The as- signed DGGE allele scores under each run time indicate relative distances of the clamped allele migration. Thus, the alleles scored as 50-65 (1 and 5) had the highest overall $\mathrm{T}_{\mathrm{m}}$ and traveled the greatest distances (to the highest concentration of denaturant) through the gel before migration was retarded by partial-strand dissociation. Conversely, allele 29, which was scored as 400 under both run times, had one of the lowest overall $\mathrm{T}_{\mathrm{m}}$ and its migration was retarded at the lowest denaturant concentration. For the homoduplex $\alpha 1$ allele sequences, increasing migration distance through the denaturing gradient was strongly correlated with increasing GC content of the fragments (Table 1 and Figure 3). However, the presence/absence of the two-codon insert, and the GC content of the last $50 \mathrm{nt}$ (distal to the GC clamp) also had apparent influence on allelic migration. Before any allele dissociation, they separated according to size, with those containing the twocodon insert migrating slower than those without the insert (data not shown). However, once alleles began to dissociate, the insert was apparently less influential on allele migration than was the GC content. For example, allele 18, which possesses both the insert and a relatively high GC content ( $48 \%$ of the variable sites), migrated as far as many of the alleles without the insert. Increased GC content distal to the clamp increased the $\mathrm{T}_{\mathrm{m}}$ of the homoduplexes more than did increased GC content close to the clamp. Alleles 1, 5, 18, 31, 8 and 23 contained the highest GC content in variable positions of last $50 \mathrm{nt}$ and consequently migrated the farthest distances on the gel. Allele 7, which lacked the insert, also had a relatively high overall GC content $(50 \%)$ but did not migrate as far, apparently as the result of a low GC content in the low melting domain and the lack of the 3-bp GC string in positions 187-189 (Figure 3 ).

Alleles that shifted (exchanged relative positions in a ladder of migration distance) were not necessarily similar to each other in nucleotide sequence and, in fact, often did not share the presence/absence of the two-codon insert. The most notable shifting allele was allele 9, which was scored as 245 at the 5.25-h run time and 225 at the 6$\mathrm{h}$ run time (Table 1). Allele 9 has a relatively low GC content in the low melting domain, a relatively high GC content in the high melting domain and lacks the two-codon insert. As such, before dissociation (at run times $<4 \mathrm{~h}$ ), allele 9 migrated relatively quickly, with the other alleles lacking the insert.

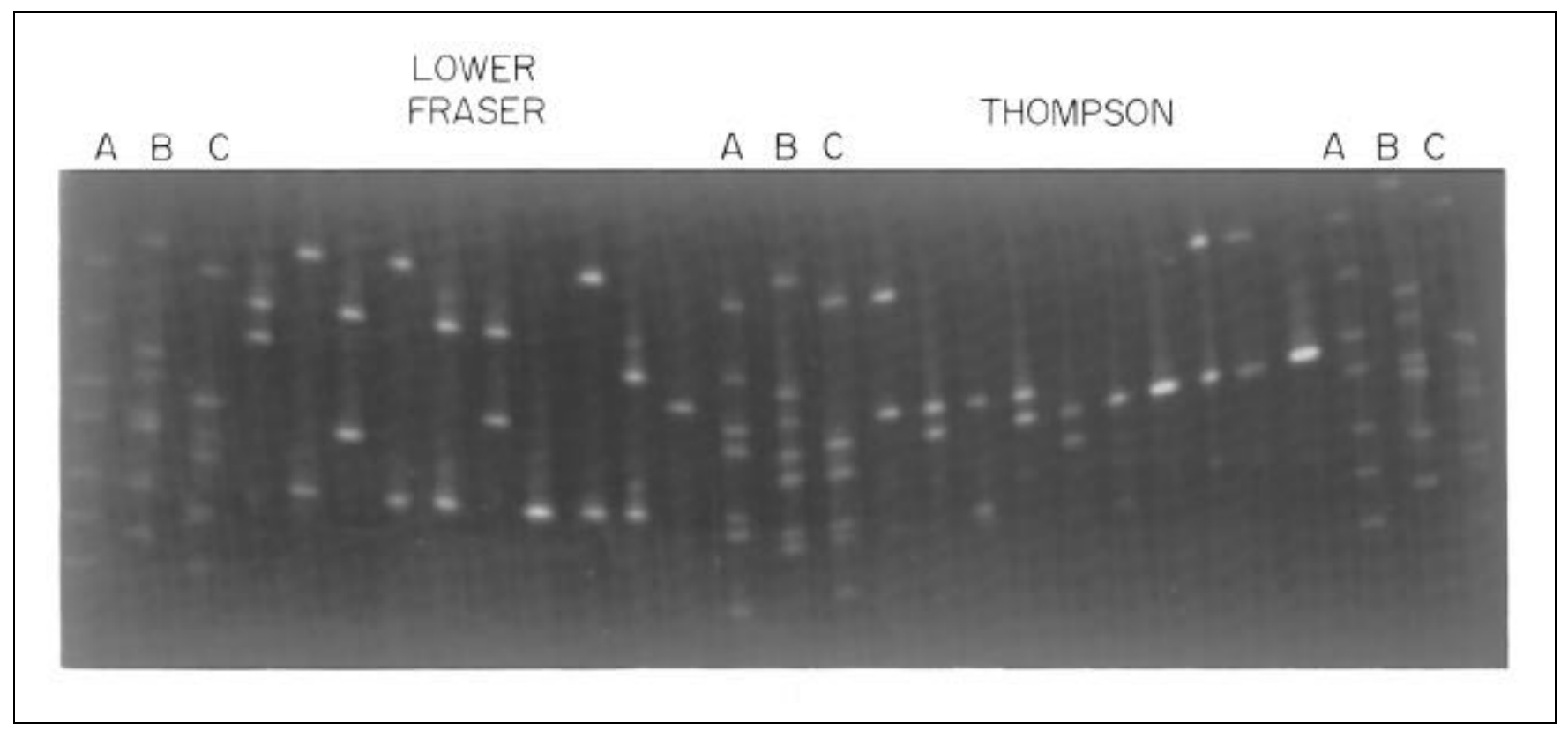

Figure 5. Parallel DGGE of chinook salmon from two populations in the Fraser River drainage in British Columbia, Canada. Control allele sets are in A, $\mathrm{B}$ and $\mathrm{C}$ lanes. Alleles 2-3-4, 13, 21, 31, 32, 33 and 36 are not included in the control allele sets, and allele 20 is present in control lanes A and C. Note the differences in allele frequencies between the two populations (10). 
However, allele 9 began to dissociate relatively early and, at a run time of $5.25 \mathrm{~h}$, it was surpassed by a number of alleles possessing the two-codon insert (alleles 27, 10 and 16) (Figure 3). At a 6-h run time, after migration into a denaturant concentration at which dissociation in the low melting domain was completed and in the high melting domain was initiated, allele 9 migrated past allele 27, which contains the twocodon insert and a lower GC content in the high melt domain. At even longer run times, allele 9 migrated farther than other adjacent alleles with the twocodon insert: alleles 10 and $16(6.5+\mathrm{h})$ and alleles 25 and 26 (>7 h; data not shown). Because allele 9 shifts so readily (it can actually shift across a single gel if the denaturing gradient is not completely even), it was difficult to score reliably at a single run time. In our initial population survey, we ran only the 6-h run time, and we combined alleles 9, 10, 16 and 27 into a single DGGE bin (10). However, with careful monitoring of the relative positions of these alleles, and scoring alleles over two run times, they can be identified separately. Allele 9 is also a useful marker for gel-to-gel run uniformity.

\section{Troubleshooting}

Several factors affected our ability to produce DGGE gels of sufficient quality to score over the six-month time period of the population survey and in developing DGGE methodology for additional MHC loci. The purity and age of the acrylamide strongly affected the resolution and migration of fragments. We found only high-purity molecular grade acrylamide produced consistent migration conditions, and acrylamide solutions containing the denaturants were only stable for as many as 7 days. Low-purity or old acrylamide produced gels with fuzzy bands and/or altered migration patterns. Brands and batches of acrylamide sometimes had an effect on migration, but one brand did not give the best results for all loci examined. We performed most of the $\alpha 1$ population survey using a single brand of acrylamide and kept track of batch numbers for problem solving (batches over six months old were sometimes problem- atic). Voltage and run time were also important. High voltage runs (>120 V) produced gel-to-gel inconsistency in the separation of alleles. Long run times $(>17 \mathrm{~h}$ ) produced fuzzy bands and poor resolution. Best results for most loci were obtained with low voltage overnight runs of not greater than $17 \mathrm{~h}$. To maximize the throughput of the D Gene (D Code) system, we ran the $\alpha 1$ analysis with daytime runs (at $120 \mathrm{~V}$ maximum) and analyzed other loci in overnight runs. Because it is difficult to get exactly the same migration distances for day and night runs (both times and voltages need to be adjusted), we analyzed each locus consistently using day or night runs.

\section{DISCUSSION}

DGGE has proven to be a rapid and sensitive technique to examine nucleotide sequence variation in large-scale population surveys. In our system, only two of our previously sequenced alleles were not detectable using DGGE, and these varied by two transversions, which are the most difficult mutations to detect because they do not alter the overall base composition of the DNA fragment (15). In addition, by resequencing all of the alleles from our original study that were not differentiated by DGGE, we discovered that some of the original sequences were artifacts. However, through our population surveys using DGGE, 12 new alleles were identified and later sequenced (10). Of the 31 presently known allele sequences, DGGE distinguishes all but one of the alleles varying by more than one nucleotide, and 4/6 sets of alleles varying by a single nucleotide. To date, we have examined over 2500 chinook salmon from 32 populations.

Because the MHC gene used contained a large number of alleles, we used the homoduplex bands, each of which corresponds to a single allele, as opposed to heteroduplex bands for allelic distinctions. However, other studies have used heteroduplex banding patterns. These have the advantage of greater sensitivity in distinguishing similar alleles, but the added difficulty in the banding patterns that must be recognized to identify each allele (two het- 
Table 1. DGGE Allele Designations

\begin{tabular}{|c|c|c|c|c|c|c|c|}
\hline Alleles & $\begin{array}{l}5.25 \mathrm{~h} \\
\text { Score }\end{array}$ & $\begin{array}{c}6 \mathrm{~h} \\
\text { Score }\end{array}$ & $\begin{array}{c}\text { Shifts } \\
\text { Position with } \\
\text { Allele(s) }\end{array}$ & $\begin{array}{l}\text { Reason } \\
\text { Binned }\end{array}$ & $\begin{array}{l}\text { Sequence } \\
\text { Variation }\end{array}$ & $\begin{array}{l}\text { 2-Codon } \\
\text { Insert }\end{array}$ & $\begin{array}{c}\text { GC } \\
\text { Content }\end{array}$ \\
\hline 1 & 50 & 65 & 1 & & & NO & 59 \\
\hline $5(6)$ & 60 & 55 & 5 & & & NO & 52 \\
\hline 18 & 100 & 100 & 31 & & & YES & 48 \\
\hline 31 & 90 & 110 & 18,36 & & & NO & 48 \\
\hline 36 & 110 & 135 & $31,8,23$ & & & NO & 45 \\
\hline 8 & 120 & 120 & 36 & & & NO & 48 \\
\hline 23 & 120 & 130 & 36,8 & & & NO & 48 \\
\hline $22(17)$ & 150 & 140 & & & & YES & 45 \\
\hline 26 & 150 & 150 & $2-3-4$ & & & YES & 45 \\
\hline $2,3,4$ & 165 & 150 & 26,11 & inseparable & $1-2 \mathrm{bp}$ & NO & 52 \\
\hline 11 & 160 & 160 & $2-3-4$ & & & YES & 45 \\
\hline 25 & 200 & 200 & & & & NO & 48 \\
\hline 10 & 210 & 210 & & & & YES & 41 \\
\hline 16 & 225 & 225 & 9 & & & YES & 45 \\
\hline 27 & 240 & 240 & 9 & & & YES & 38 \\
\hline 9 & 245 & 225 & 16,27 & & & NO & 45 \\
\hline 12,13 & 270 & 270 & & run similarly $(12,13)$ & $1 \mathrm{bp}$ & YES & $39-41$ \\
\hline \multirow[t]{2}{*}{28} & & & & run similarly $(13,28)$ & $2 \mathrm{bp}$ & & \\
\hline & & & & inseparable $(12,28)$ & $1 \mathrm{bp}$ & & \\
\hline 14 & 270 & 280 & $12-13-28,21$ & & & YES & 41 \\
\hline 21 & 280 & 280 & 14,15 & & & YES & 41 \\
\hline 15 & 280 & 285 & 21 & & & YES & 38 \\
\hline 35 & 290 & 295 & 7 & & & YES & 35 \\
\hline 7 & 295 & 295 & 35 & & & NO & 48 \\
\hline 30 & 320 & 320 & & & & YES & 41 \\
\hline 33 & 340 & 340 & & & & YES & 41 \\
\hline $20(19)$ & 350 & 350 & 32 & & & YES & 38 \\
\hline 32 & 350 & 345 & 20 & & & NO & 45 \\
\hline 29 & 400 & 400 & & & & NO & 38 \\
\hline total & 22 & 23 & & & & & \\
\hline \multicolumn{8}{|c|}{$\begin{array}{l}\text { "Sequenced alleles" } 1-22 \text { were obtained in a sequencing study (13), while alleles } 23-36 \text { (no } 34 \text { ) were resolved using DGGE } \\
\text { (10). Alleles } 17,20 \text { and } 6 \text { (13) (in brackets) have been reclassified as other existing alleles. DGGE allele numbers reflect the } \\
\text { relative position of the alleles run under the optimal DGGE conditions ( } 5.25 \text { and } 6 \mathrm{~h} \text { at } 120 \mathrm{~V} \text { ) noted. Sequencing alleles were } \\
\text { binned into DGGE alleles when alleles run similarly or are inseparable under both DGGE run times. Alleles that shift relative } \\
\text { position between the two run times are noted. The amount of sequence variation between alleles within each "DGGE allele" } \\
\text { bin is also noted. Total indicates the total number of alleles differentiated at each run time. }\end{array}$} \\
\hline
\end{tabular}

eroduplex bands for heterozygous individuals, six heteroduplex bands if heteroduplexes are generated by the addition of a reference allele) $(4,14)$. Although the regular scoring of all 31 alleles based on heteroduplex banding patterns is not practical, heteroduplex analysis in DGGE could be used as an additional step to identify alleles that are indistinguishable or too similar to score separately as homoduplexes (e.g., alleles 2, 3 and 4; alleles 12, 28 and 13).
We have also conducted surveys of coho salmon populations at the $\alpha 1$ exon described and the $\alpha 2$ exon of the same locus using DGGE (11). With limited $\alpha 1$ and $\alpha 2$ sequence information for coho salmon, we used DGGE to identi- 
fy alleles for our standards. For each exon, the melting curves were obtained by perpendicular gradient gels run on a few clones of known allele sequence. The overall melting range was then used as the initial gradient on parallel gels, and approximately 30 individuals representing a broad geographic range throughout British Columbia were surveyed in a time series over 5 gels. All of the alleles differentiated in the time series were then amplified from genomic DNA and combined to create allele standards as described. The optimal running conditions for $\alpha 1$ in coho salmon were quite similar to those for chinook salmon, though some refinements were made through a time series analysis. Our population analysis at the $\alpha 1$ and $\alpha 2$ exons in coho salmon actually began before the sequences were determined. New alleles discovered during the population surveys were quickly added to the standards. To date, we have examined $\alpha 1$ allelic variation in over 5500 coho salmon from 60 populations and $\alpha 2$ allelic variation in over 12000 coho salmon from 82 populations.

DGGE has been used for population analysis in only a limited number of studies $(2,17)$, mostly to identify haplotypes among a few individuals for sequencing. Our study offers the first example of the potential use of DGGE for large-scale population surveys of highly polymorphic DNA loci $(10,11)$. In our laboratory, one person can analyze 160 individuals on DGGE (at a single run time) per day, and our D Gene and $\mathrm{D}$ Code gel rigs are used for both day ( $\alpha 1$ exon) and night ( $\alpha 2$ exon) runs. This rapid rate of analysis of nucleotide sequence variation allows us to quickly accumulate large databases and to use DGGE for applied, real-time fisheries management issues such as stock identification in mixed stock fisheries, escapement enumeration within river sys- tems and the identification of escaped, farmed fish.

The DGGE methodology was initially designed as a sensitive technique to detect single-base mutations $(3,19)$. Recently, researchers have begun using DGGE as a tool to separate highly variable alleles for identification through direct sequencing $(1,9,11)$. Other studies have used DGGE to compare phylogenic assemblages of bacterioplankton (14). Our study is the first to use DGGE to differentiate and directly identify highly variable alleles in comparison with allele standards. However, because the $\alpha 1$ allele sequences vary to a great extent over multiple melting domains, and possibly because we carefully monitored allele positions over many gels and through a time series analysis, we encountered more problems with allelic shifts in migration than noted in previous studies. These problems were resolved by using a sys- 
tem of standards, so that almost all alleles with any level of sequence variation were accurately and reliably discriminated. For fragments with a single melting domain (e.g., the salmonid MHC class I $\alpha 2$ exon that we survey in coho salmon), only a single run time is necessary to differentiate alleles.

Temperature gradient gel electrophoresis (TGGE) and constant denaturant gel electrophoresis (CDGE) are methodologies related to DGGE. The chemical gradient used in DGGE is replaced by a temperature gradient in TGGE. We tested TGGE (using the D Code system) on the chinook $\alpha 1$ alleles and were unable to differentiate as many alleles as we did with DGGE, nor achieved the same band "crispness". TGGE has been successfully used in other population studies to resolve small numbers of alleles (4). CDGE separates fragments at a single denaturant concentration and can be useful when there is only a single melting domain and few alleles or when multiple gels, each at different concentrations of denaturant, are used. Because we knew the chinook salmon $\alpha 1$ alleles contained two melting domains, this technique was not tested.

The DGGE methodology described can be used on any region of DNA with nucleotide sequence variation. Best results are obtained with fragments of $300-500 \mathrm{bp}$, but larger fragments can be analyzed by first digesting them with restriction endonucleases (19). In DGGE, the detection of length variation is often superseded by nucleotide variation, but DGGE could be an invaluable tool for detecting homoplasy in microsatellite alleles. The use of DGGE virtually eliminates the need to sequence large numbers of clones to examine sequence variability. However, as some sequences may not be detectable under a single run condition, it is generally prudent to sequence two or three clones for each of the fragments differentiated by DGGE or to run a time series on fragments with similar migration behavior. Direct sequencing of fragments cut out of DGGE gels can further expedite the process (9). Finally, by careful use of standard networks of known alleles, DGGE is a highly sensitive and expeditious technique for detecting nucleotide sequence variation in large-scale population surveys.

\section{ACKNOWLEDGMENTS}

We appreciate the technical assistance provided by L. Dube. This work was supported in part by the Department of External Affairs (Japan Science and Technology Fund [JSTF] Grant No. PNR-2691) and by the Department of Fisheries and Oceans.

\section{REFERENCES}

1.Aldridge, B.M., S.M. McGuirk, R.J. Clark, L.A. Knapp, D.I. Watkins and D.P. Lunn. 1998. Denaturing gradient gel electrophoresis: a rapid method of differentiating BoLA-DRB3 alleles. Anim. Genet. 29:389-394.

2.Brown, J.M., J.H. Leebens-Mack, J.N. Thompson, O. Pellmyr and R.G. Harrison. 1997. Phylogeography and host association in a pollinating seed parasite Greya politella (Lepidoptera: Prodoxidae). Mol. Ecol. 6:215224.

3.Fischer, S.G. and L.S. Lerman. 1983. DNA fragments differing by single base-pair substitutions are separated in denaturing gradient gels: Correspondence with melting theory. Proc. Natl. Acad. Sci. USA 80:1579-1583.

4.Fuller, S.J., J.C. Wilson and P.B. Mather. 1997. Patterns of differentiation among wild rabbit populations Oryctolagus cuniculus L. in arid and semiarid ecosystems of north-eastern Australia. Mol. Ecol. 6:145-153.

5.Greiner, T.C., M. Raffeld, C. Lutz, F. Dick and E.S. Jaffe. Analysis of T cell receptor- $\gamma$ gene rearrangements by denaturing gradient gel electrophoresis of GC-clamped polymerase chain reaction products. Am. J. Pathol. 146:4655.

6.Grimholt, U., I. Hordvik, V.M. Fosse, I. OIsaker, C. Endresen and O. Lie. 1993. Molecular cloning of major histocompatibility complex class I cDNA's in Atlantic salmon (Salmo salar). Immunogenetics 37:469-473.

7.Hayashi, K. 1991. PCR-SSCP: A simple and sensitive method for detection of mutations in the genomic DNA. PCR Methods Appl. 1:3438 .

8.Hughes, A.L. 1991. MHC polymorphism and the design of captive breeding programs. Conserv. Biol. 5:29-251.

9.Knapp, L.A., L.F. Cadavid, M.E. Eberle, S.J. Knechtle, R.E. Bontrop and D.I. Watkins. 1997. Identification of new mamu-DRB alleles using DGGE and direct sequencing. Immunogenetics 45:171-179.

10.Miller, K.M. and R.E. Withler. 1997. Mhe diversity in Pacific salmon: Population structure and trans-species allelism. Hereditas 127:8395.

11.Miller, K.M. and R.E. Withler. 1998. The salmonid class I MHC: limited diversity in a primitive teleost. Immunol. Rev. 166 (In Press).

12.Miller, K.M., R.E. Withler and T.D. Beacham. 1996. Stock identification of coho salmon (Oncorynchus kisutch) using minisatellite DNA variation. Can. J. Fish. Aquat. Sci. 53:181-195.
13.Miller, K.M., R.E. Withler and T.D. Beacham. 1997. Molecular evolution at Mhc genes in two populations of chinook salmon Oncorhynchus tshawytscha. Mol. Ecol. 6:937954.

14.Murray, A.E., J.T. Hollibaugh and C. Orrego. 1996. Phylogenetic compositions of bacterioplankton from two California estuaries compared by denaturing gradient gel electrophoresis of $16 \mathrm{~S}$ rDNA fragments. Appl. Environ. Microbiol. 62:2676-2680.

15.Myers, R.M., S.G. Fischer, L.S. Lerman and T. Maniatis. 1985. Nearly all single base substitutions in DNA fragments joined to a GCclamp can be detected by denaturing gradient gel electrophoresis. Nucleic Acids Res. 13:3131-3145

16.Nei, M. and A.L. Hughes. 1991. Polymorphism and the evolution of the major histocompatibility complex loci in mammals, p. 222247. In R.K. Slander, A.G. Clark and T.S. Whittam (Eds.), Evolution at the Molecular Level. Sinauer Associates, Sunderland, MA.

17.Norman, J.A., C. Moritz and C.J. Limpus. 1994. Mitochondrial DNA control region polymorphisms: genetic markers for ecological studies of marine turtles. Mol. Ecol. 3:363-373.

18.O'Brien, S.J., M.E. Roelke, L. Marker, A. Newmen, C.A. Winkler, D. Meltzer, L. Colly, J.F. Evermann et al. 1985. Genetic basis for species vulnerability in the cheetah. Science 227:1428-1434.

19.Sheffield, V.C., D.R. Cox, L.S. Lerman and R.M. Myers. 1989. Attachment of a 40-basepair $\mathrm{G}+\mathrm{C}$-rich sequence (GC-clamp) to genomic DNA fragments by the polymerase chain reaction results in improved detection of single-base changes. Proc. Natl. Acad. Sci. USA 86:232-236.

20.Singh, N., S. Agrawal and A.K. Rastogi. 1997. Infectious diseases and immunity: special reference to major histocompatibility complex. Emerg. Infect. Dis. 3:41-49.

21.Small, M.P., T.D. Beacham, R.E. Withler and R.J. Nelson. 1998. Discrimination of coho salmon (Oncorhynchus kisutch) populations within the Fraser River, British Columbia using microsatellite DNA markers. Mol. Ecol. 7:141155.

22.Valero, M.C., E. Velasco, F. Moreno and C. Hernandez-Chico. 1994. Characterization of four mutations in the neurofibromatosis type 1 gene by denaturing gradient gel electrophoresis (DGGE). Hum. Mol. Genet. 3:639-641.

Received 13 April 1999; accepted 6 July 1999.

\section{Address correspondence to:}

Kristina M. Miller

Pacific Biological Station

Department of Fisheries and Oceans

Nanaimo BC V9R 5K6, Canada

Internet:millerk@dfo-mpo.gc.ca 